\title{
WeAK Hopf ALGEBras CORRESPONDing to CARTAN MATRICES
}

\author{
Shilin Yang* \\ College of Applied Sciences, Beijing University of Technology \\ Beijing 100022, P. R. China \\ slyang@bjut.edu.cn
}

\begin{abstract}
We replace the group of group-like elements of the quantized enveloping algebra $U_{q}(\mathfrak{g})$ of a finite dimensional semisimple Lie algebra $\mathfrak{g}$ by some regular monoid and get the weak Hopf algebra $\mathfrak{w}_{q}^{\mathrm{d}}(\mathfrak{g})$. It is a new subclass of weak Hopf algebras but not Hopf algebras. Then we devote to constructing a basis of $\mathfrak{w}_{q}^{d}(\mathfrak{g})$ and determine the group of weak Hopf algebra automorphisms of $\mathfrak{w}_{q}^{\mathrm{d}}(\mathfrak{g})$ when $q$ is not a root of unity.
\end{abstract}

\section{Introduction}

Recently, many mathematicians are interested in generalizations of Hopf algebras, of which importance has been recognized in both mathematics and physics. One way is to introduce the notion of a weak co-product, such that $\Delta(1) \neq 1 \otimes 1$, which was motivated by the study of symmetries in low dimensional quantum field theory. This resulted in the definition of weak Hopf algebras, introduced by Böhm, Nill, and Szlachányi (see for example [3]). Since they are not bi-algebras, but almost bi-algebras, there were also axioms required to define a weak antipode, differing slightly from the usual notion of a Hopf algebra. The face algebras [7] and generalized Kac algebras [19] are examples of this class of weak Hopf algebras.

It is possible to define a weak antipode on a given bi-algebra. This was introduced by Li in [11]. By definition, a bialgebra $\mathcal{H}=(H, \mu, \eta, \Delta, \varepsilon)$ over a field $k$ together with the identity map id in $\operatorname{hom}_{k}(H, H)$ is called a weak Hopf algebra if there exists $T \in \operatorname{hom}_{k}(H, H)$ such that id $* T * \mathrm{id}=\mathrm{id}$ and $T * \mathrm{id} * T=T$ where $*$ is the convolution product. The map $T$ is called a weak antipode. It is noted that the notion of Hopf algebras, and left or right Hopf algebras are included in this class of weak Hopf algebras (see [17, 16, 6]). Another typical example is the weak quantum algebras $\mathfrak{w s l}_{q}(2)$ and $v \mathfrak{s l}_{q}(2)$ constructed in [12]. It is the generalization by replacing the set of group-like elements of $U_{q}\left(\mathfrak{s l}_{2}\right)$ by the set of some regular monoid, where $U_{q}\left(\mathfrak{s l}_{2}\right)$ is the quantized enveloping algebra corresponding to 3-dimensional semisimple Lie algebra. The basis and some properties of $\mathfrak{w s l}_{q}(2)$ (resp.

${ }^{*}$ The author is partially supported by the National Science Foundation of China (Grant. 10271014) and the Fund of Elitist Development of Beijing City (Grant: 20042D0501518). 
$\left.v \mathfrak{s l}_{q}(2)\right)$ were studied in [12]. Recently, Aizawa and Isaac [2] gave a description of weak Hopf algebra $\mathfrak{w s l}_{q}^{d}(n)$ in general, which is corresponding to the known Hopf algebra $U_{q}\left(\mathfrak{s l}_{n}\right)$.

Our aim is to give more non-trivial examples for weak Hopf algebras in the sense of Li. Following the idea $[2,12]$, we would like to extend this construction to the more general one $\mathfrak{w}_{q}^{d}(\mathfrak{g})$ corresponding to arbitrary finite dimensional semisimple Lie algebra $\mathfrak{g}$.

Thanks to the definition of quantum group $U_{q}(\mathfrak{g})$ defined by $[13,10]$, we can also replace the group $G\left(U_{q}(\mathfrak{g})\right)$ of group-like elements by some regular monoid and get the weak Hopf algebra $\mathfrak{w}_{q}^{\mathrm{d}}(\mathfrak{g})$, which is resulted from quantum group $U_{q}(\mathfrak{g})[12,10,13]$. This successful construction provides us a new subclass of weak Hopf algebras but not Hopf algebras. As does the classic quantum group $U_{q}(\mathfrak{g})$, we will determine the basis and the group of weak Hopf algebra automorphisms of $\mathfrak{w}_{q}^{\mathrm{d}}(\mathfrak{g})$.

To determine the basis of $\mathfrak{w}_{q}^{\mathrm{d}}(\mathfrak{g})$, we first show that $\mathfrak{w}_{q}^{\mathrm{d}}(\mathfrak{g})$ can be written as a direct sum of its two ideals and one of them is just isomorphic to the classic quantum group $U_{q}(\mathfrak{g})$. Then we apply the PBW Theorem for $U_{q}(\mathfrak{g})$ to describe a basis of $\mathfrak{w}_{q}^{\mathrm{d}}(\mathfrak{g})$. If $q$ is not a root of unity, the group of Hopf algebra automorphisms of $U_{q}(\mathfrak{g})$ was determined in [4]. The case when $q$ is a root of unity, it was considered in the recent work [15]. In the present paper, we will determine the group of weak Hopf algebra automorphisms of $\mathfrak{w}_{q}^{\mathrm{d}}(\mathfrak{g})$ under the condition that $q$ is not a root of unity. The method is to apply the result of $[4$, Corollary 4.3] and some technical lemmas.

The paper is organized as follows.

In Sec. II, we give some notations and the definition of weak Hopf algebra $\mathfrak{w}_{q}^{\mathrm{d}}(\mathfrak{g})$. The ideal to construct the algebra $\mathfrak{w}_{q}^{\mathrm{d}}(\mathfrak{g})$ and some basic properties are described. In Sec. III, we give the comultiplication of $\mathfrak{w}_{q}^{\mathrm{d}}(\mathfrak{g})$ in order that it is a weak hopf algebra but not a Hopf alegbra. The proof somewhat is basic and direct. In Sec. IV, we describe the basis of $\mathfrak{w}_{q}^{\mathrm{d}}(\mathfrak{g})$ by the techonique of Lusztig's constructing PBW basis of $U_{q}(\mathfrak{g})$. In the final section, we study and determine the group of Hopf automorphisms of $U_{q}(\mathfrak{g})$.

\section{$2 \quad$ Weak quantum algebras $\mathfrak{w}_{q}^{\mathbf{d}}(\mathfrak{g})$}

In this paper, we always assume that $k$ is a field of characteristic 0 . Let $\mathfrak{g}$ be a finite dimensional semisimple Lie algebra. For the simplicity, we can assume that $\mathfrak{g}$ is also simple. Then there is a finite positive symmetrizable Cartan matrix $\mathcal{C}=\left(a_{i j}\right)_{n \times n}$ corresponding to it (see [8]).

Now we let $R$ be the root system of $\mathfrak{g}$ and we fix a basis $I=\left\{\alpha_{1}, \cdots, \alpha_{n}\right\}$ of $R$. Let $W$ be the Weyl group of $R$. It is well known that there is a unique $W$-invariant scalar product $($,$) on the vector space generated by R$ over the reals such that $(\alpha, \alpha)=2$ for all short roots $\alpha$ in $R$. Set for each $\alpha_{i} \in I, 1 \leq i \leq n$

$$
d_{i}=\frac{\left(\alpha_{i}, \alpha_{i}\right)}{2} \text {. }
$$

It is noted that $\left(\alpha_{i}, \alpha_{j}\right)=d_{i} a_{i j}=d_{j} a_{j i}$.

Let $q \in k$ and $q_{i}=q^{d_{i}}, 1 \leq i \leq n$. It is assumed that $q_{i} \neq \pm 1,0$ for all $i$. For an 
indeterminate $x$ and an integer $m$, let

$$
[m]_{x}=\frac{x^{m}-x^{-m}}{x-x^{-1}},[m] !_{x}=[m]_{x} \cdots[1]_{x},[0]_{x} !=1,
$$

and

$$
\left[\begin{array}{c}
m \\
s
\end{array}\right]_{x}=\frac{[m] !_{x}}{[s] !_{x}[m-s] !_{x}}
$$

One can review the definition of the quantized enveloping algebra $U_{q}=U_{q}(\mathfrak{g})$ by referring to $[9,10,13]$. For the completeness, we describe it here as follows. The algebra $U_{q}(\mathfrak{g})$ generated by $4 n$ generators $e_{i}, f_{i}, k_{i}, k_{i}^{-1}(1 \leq i \leq n)$ with the relations

$$
\begin{aligned}
& k_{i} k_{j}=k_{j} k_{i}, k_{i} k_{i}^{-1}=k_{i}^{-1} k_{i}=1 \\
& k_{i} e_{j} k_{i}^{-1}=q_{i}^{a_{i j}} e_{j}, k_{i} f_{j} k_{i}^{-1}=q_{i}^{-a_{i j}} f_{j} ; \\
& e_{i} f_{j}-f_{j} e_{i}=\delta_{i j} \frac{k_{i}-k_{i}^{-1}}{q_{i}-q_{i}^{-1}} ; \\
& \sum_{s=0}^{1-a_{i j}}(-1)^{s}\left[\begin{array}{c}
1-a_{i j} \\
s
\end{array}\right]_{q_{i}} e_{i}^{1-a_{i j}-s} e_{j} e_{i}^{s}=0, \text { if } i \neq j ; \\
& \sum_{s=0}^{1-a_{i j}}(-1)^{s}\left[\begin{array}{c}
1-a_{i j} \\
s
\end{array}\right]_{q_{i}} f_{i}^{1-a_{i j}-s} f_{j} f_{i}^{s}=0, \text { if } i \neq j .
\end{aligned}
$$

To generalize the invertibility condition (2.1), one way is to weak the invertibility to regularity, in which instead of $\left\{k_{i}, k_{i}^{-1}\right\}$ by a pair $\left\{K_{i}, \bar{K}_{i}\right\}$ for all $1 \leq i \leq n$ subjecting to some relations. For example, we can introduce a projector $J$ such that

$$
\begin{aligned}
& J=K_{i} \bar{K}_{i}=\bar{K}_{i} K_{i}, \\
& J K_{i}=K_{i}, \quad \bar{K}_{i} J=\bar{K}_{i}, \\
& J \bar{K}_{i}=\bar{K}_{i}, \quad K_{i} J=K_{i} .
\end{aligned}
$$

for $1 \leq i \leq n$. To generalize other relations of definition, we need some terminologies for simplicity. For example, if $E_{i}$ satisfies

$$
K_{j} E_{i}=q_{i}^{a_{i j}} E_{i} K_{j}, E_{i} \bar{K}_{j}=q_{i}^{a_{i j}} \bar{K}_{j} E_{i}, \forall j,
$$

we say $E_{i}$ is type 1 . Moreover, if $E_{i}$ satisfies

$$
K_{j} E_{i} \bar{K}_{j}=q_{i}^{a_{i j}} E_{i}, \forall j
$$

we say $E_{i}$ is type 2 . The same convention holds for $F_{i}$ by replacing $E_{i}$ with $F_{i}$ and $a_{i j}$ with $-a_{i j}$ in the above relations.

We borrow some notations from the reference [2], $2 n$ simple generators $E_{i}$ and $F_{i}$ are listed by starting with the $E_{i}$ followed by the $F_{i}$, where a 1 is to indicate the use of a type 1 generator and a 0 is to the use of a type 2 generator. Then we write down a list of 0 's and 1's in the order corresponding to the generators determined by their type. This gives a sequence $\mathrm{d}$ contained 0 and 1 in binary representation of length $2 n$. It is noted that $d$ 
contains all the information on the relations with the generators $E_{i}$ and $F_{j}$, all $K_{j} / \bar{K}_{j}$, and $J$. We write d in terms of its binary expansion

$$
\mathrm{d}=\left(\kappa_{1}, \cdots, \kappa_{n} \mid \bar{\kappa}_{1}, \cdots, \bar{\kappa}_{n}\right),
$$

where the bar separates the values representing the $E_{i}$ and $F_{i}$, and where the $\kappa_{i}$ and $\bar{\kappa}_{i}$ have values of either 0 or 1 . Accordingly, we can say $E_{i}$ and $F_{i}, \alpha_{i} \in I$ are type $\mathrm{d}$ in an obvious sense.

Now we can write down this generalization explicitly as follows.

Definition 2.1 The algebra $\mathfrak{w}_{q}^{d}(\mathfrak{g})$ is generated by the $4 n+1$ variables $E_{i}, F_{i}, K_{i}, \bar{K}_{i}(1 \leq$ $i \leq n)$ and $J$ with the relations: for all $1 \leq i, j \leq n$,

$$
\begin{aligned}
& J=K_{i} \bar{K}_{i}, \\
& K_{i} \bar{K}_{j}=\bar{K}_{j} K_{i}, K_{i} K_{j}=K_{j} K_{i}, \bar{K}_{i} \bar{K}_{j}=\bar{K}_{j} \bar{K}_{i} ; \\
& J K_{i}=K_{i}, \quad J \bar{K}_{i}=\bar{K}_{i}, \\
& E_{i}, F_{i} \text { are type d, } \\
& E_{i} F_{j}-F_{j} E_{i}=\delta_{i j} \frac{K_{i}-\bar{K}_{i}}{q_{i}-q_{i}^{-1}} ; \\
& \sum_{s=0}^{1-a_{i j}}(-1)^{s}\left[\begin{array}{c}
1-a_{i j} \\
s
\end{array}\right]_{q_{i}} E_{i}^{1-a_{i j}-s} E_{j} E_{i}^{s}=0, \text { if } i \neq j ; \\
& \sum_{s=0}^{1-a_{i j}}(-1)^{s}\left[\begin{array}{c}
1-a_{i j} \\
s
\end{array}\right]_{q_{i}} F_{i}^{1-a_{i j}-s} F_{j} F_{i}^{s}=0, \text { if } i \neq j,
\end{aligned}
$$

The algebra $\mathfrak{w}_{q}^{d}(\mathfrak{g})$ is said to be a d-type weak quantum algebra associated to Lie algebra g.

It is easy to see that there are $2^{2 n}$ possible weak quantum algebras $\mathfrak{w}_{q}^{\mathrm{d}}(\mathfrak{g})$ corresponding to the sequence $d$ in total.

It is easy to see that (2.9)-(2.10) generalize the relation (2.1), and the relations (2.11)(2.12) generalize the corresponding relations $(2.2)-(2.3)$. The notations $P_{i}(1 \leq i \leq n)$ are defined by

$$
P_{i}^{k}= \begin{cases}K_{i}^{k}, & k>0 \\ J, & k=0 \\ \bar{K}_{i}{ }^{k}, & k<0\end{cases}
$$

It is easy to see that $P_{i}^{k}$ satisfy the regularity conditions

$$
P_{i}^{k} P_{i}^{-k} P_{i}^{k}=P_{t}^{k}
$$

for all $k \in \mathbb{Z}$.

There are some properties for $\mathfrak{w}_{q}^{\mathrm{d}}(\mathfrak{g})$ which are used later.

Lemma 2.2 The idempotent $J$ is in the centre of $\mathfrak{w}_{q}^{d}(\mathfrak{g})$. 
Proof. Indeed, for $K_{j}$ and $\bar{K}_{j}$, it follows from (2.9) and (2.10). For instance,

$$
K_{j} J=K_{j} K_{j} \bar{K}_{j}=K_{j} \bar{K}_{j} K_{j}=J K_{j}
$$

For $E_{j}$, if it is type 1 , then we have

$$
J E_{j}=K_{i} \bar{K}_{i} E_{j}=q_{i}^{-a_{i j}} K_{i} E_{j} \bar{K}_{i}=E_{j} J
$$

by (2.6); if $E_{i}$ is type 2 ,

$$
J E_{i}=K_{i} \bar{K}_{i} E_{i}=q_{i}^{-2} K_{i} \bar{K}_{i} K_{i} E_{i} \bar{K}_{i}=q_{i}^{-2} K_{i} E_{i} \bar{K}_{i}=q_{i}^{-2} K_{i} E_{i} \bar{K}_{i} K_{i} \bar{K}_{i}=E_{i} J
$$

by (2.7). Hence, $J E_{i}=E_{i} J$ for all $i=1,2, \cdots, n$. The same argument for $F_{i}$ by $(2.11)$.

If $E_{i}$ is type 2 , hence $K_{j} E_{i} \bar{K}_{j}=q_{i}^{a_{i j}} E_{i}$ for all $1 \leq j \leq n$, then

$$
K_{j} E_{i}=K_{j} J E_{i}=K_{j} E_{i} J=K_{j} E_{i} \bar{K}_{j} K_{j}=q_{i}^{a_{i j}} E_{i} K_{j}
$$

and $E_{i} \bar{K}_{j}=q_{i}^{a_{i j}} \bar{K}_{j} E_{i}$. Similarly, if $F_{i}$ is type 2, we have

$$
K_{j} F_{i}=q_{i}^{-a_{i j}} F_{i} K_{j}, \quad F_{i} \bar{K}_{j}=q_{i}^{-a_{i j}} \bar{K}_{j} F_{i}
$$

Now, it is straightforward to check by induction that $E_{i}$ (resp. $\left.F_{i}\right), 1 \leq i \leq n$, is either type 1 or type 2 , the following relations hold in $\mathfrak{w}_{q}^{\mathrm{d}}(\mathfrak{g})$ :

$$
\begin{aligned}
& E_{i}^{m} K_{j}^{n}=q_{i}^{-m n a_{i j}} K_{i}^{n} E_{i}^{m}, \quad F_{i}^{m} K_{j}^{n}=q_{i}^{m n a_{i j}} K_{j}^{n} F_{i}^{m}, \\
& E_{i}^{m} \bar{K}_{j}^{n}=q_{i}^{m n a_{i j}} \bar{K}_{j}^{n} E_{i}^{m}, \quad F_{i}^{m} \bar{K}_{j}^{n}=q_{i}^{-m n a_{i j}} \bar{K}_{j}^{n} F_{i}^{m} .
\end{aligned}
$$

In particular, we have

$$
\begin{aligned}
& E_{i}^{m} K_{i}^{n}=q_{i}^{-2 m n} K_{i}^{n} E_{i}^{m}, \quad F_{i}^{m} K_{i}^{n}=q_{i}^{2 m n} K_{i}^{n} F_{i}^{m}, \\
& E_{i}^{m} \bar{K}_{i}^{n}=q_{i}^{2 m n} \bar{K}_{i}^{n} E_{i}^{m}, \quad F_{i}^{m} \bar{K}_{i}^{n}=q_{i}^{-2 m n} \bar{K}_{i}^{n} F_{i}^{m} .
\end{aligned}
$$

\section{The weak Hopf algebra structure of $\mathfrak{w}_{q}^{\mathbf{d}}(\mathfrak{g})$}

To make the d-type weak quantum algebra $\mathfrak{w}_{q}^{\mathrm{d}}(\mathfrak{g})$ be a weak Hopf algebra, we define three maps

$$
\begin{aligned}
& \Delta: \mathfrak{w}_{q}^{\mathrm{d}}(\mathfrak{g}) \rightarrow \mathfrak{w}_{q}^{\mathrm{d}}(\mathfrak{g}) \otimes \mathfrak{w}_{q}^{\mathrm{d}}(\mathfrak{g}) \\
& \varepsilon: \mathfrak{w}_{q}^{\mathrm{d}}(\mathfrak{g}) \rightarrow k \\
& T: \mathfrak{w}_{q}^{\mathrm{d}}(\mathfrak{g}) \rightarrow \mathfrak{w}_{q}^{\mathrm{d}}(\mathfrak{g})
\end{aligned}
$$


as follows:

$$
\begin{aligned}
& \Delta\left(K_{i}\right)=K_{i} \otimes K_{i}, \\
& \Delta\left(\bar{K}_{i}\right)=\bar{K}_{i} \otimes \bar{K}_{i}, \\
& \Delta(J)=J \otimes J, \\
& \Delta\left(E_{i}\right)= \begin{cases}1 \otimes E_{i}+E_{i} \otimes K_{i}, & E_{i} \text { is type } 1 ; \\
J \otimes E_{i}+E_{i} \otimes K_{i}, & E_{i} \text { is type } 2\end{cases} \\
& \Delta\left(F_{i}\right)= \begin{cases}F_{i} \otimes 1+\bar{K}_{i} \otimes F_{i}, & F_{i} \text { is type } 1 ; \\
F_{i} \otimes J+\bar{K}_{i} \otimes F_{i}, & F_{i} \text { is type } 2,\end{cases} \\
& \varepsilon\left(E_{i}\right)=\varepsilon\left(F_{i}\right)=0, \varepsilon\left(K_{i}\right)=\varepsilon\left(\bar{K}_{i}\right)=1, \varepsilon(J)=1,
\end{aligned}
$$

while the map $T$ has the form

$$
\begin{aligned}
T(1) & =1 \\
T\left(E_{i}\right) & =-E_{i} \bar{K}_{i}, \\
T_{i}\left(F_{i}\right) & =-K_{i} F_{i} \\
T\left(K_{i}\right) & =\bar{K}_{i} \\
T\left(\bar{K}_{i}\right) & =K_{i}, T(J)=J .
\end{aligned}
$$

Then we extend them to the whole $\mathfrak{w}_{q}^{\mathrm{d}}(\mathfrak{g})$.

In $\left[2, \operatorname{Sec}\right.$ IV], the authors investigated the algebra $\mathfrak{w} \mathfrak{s l}_{q}^{\mathrm{d}}(n)$ and claimed that $\mathfrak{w s l}_{q}^{\mathrm{d}}(n)$ is a weak Hopf algebra, of which no proof was given. In general, we yield that

Theorem 3.1 ([2, Sec. IV]) $\mathfrak{w}_{q}^{d}(\mathfrak{g})$ is a non-commutative and non-cocommutative weak Hopf algebra with the weak antipode $T$, but not a Hopf algebra.

In [2], the authors gave a classification in some sense of weak Hopf algebras corresponding to $U_{q}\left(\mathfrak{s l}_{n}\right)$. Similarly, we can follow the idea [2] to describe the isomorphism classes of weak Hopf algebras $\mathfrak{w}_{q}^{\mathrm{d}}(\mathfrak{g})$. As a consequence, we have a lot of new non-trivial examples of weak Hopf algebras for various sequences $d$.

The theorem follows from Lemma 3.2 and Lemma 3.3 below.

Lemma $3.2 \mathfrak{w}_{q}^{d}(\mathfrak{g})$ is a bialgebra with comultiplication $\Delta$ and counit $\varepsilon$. 
Proof. It can be shown by direct calculation that the following relations hold.

$$
\begin{aligned}
\Delta\left(K_{i}\right) \Delta\left(\bar{K}_{j}\right) & =\Delta\left(\bar{K}_{j}\right) \Delta\left(K_{i}\right), \\
\Delta(J) & =\Delta\left(K_{i}\right) \Delta\left(\bar{K}_{i}\right), \\
\Delta(J) \Delta\left(K_{i}\right) & =\Delta\left(K_{i}\right), \\
\Delta(J) \Delta\left(\bar{K}_{i}\right) & =\Delta\left(\bar{K}_{i}\right), \\
\varepsilon\left(K_{i}\right) \varepsilon\left(\bar{K}_{j}\right) & =\varepsilon\left(\bar{K}_{i}\right) \varepsilon\left(K_{j}\right), \\
\varepsilon(J) \varepsilon\left(K_{i}\right) & =\varepsilon\left(K_{i}\right), \\
\varepsilon(J) \varepsilon\left(\bar{K}_{i}\right) & =\varepsilon\left(\bar{K}_{i}\right), \\
\varepsilon\left(\bar{K}_{i}\right) \varepsilon\left(E_{i}\right) & =q^{-2} \varepsilon\left(E_{i}\right) \varepsilon\left(\bar{K}_{i}\right), \\
\varepsilon\left(\bar{K}_{i}\right) \varepsilon\left(F_{i}\right) & =q^{2} \varepsilon\left(F_{i}\right) \varepsilon\left(\bar{K}_{i}\right), \\
\varepsilon\left(E_{i}\right) \varepsilon\left(F_{j}\right)-\varepsilon\left(F_{j}\right) \varepsilon\left(E_{i}\right) & =\delta_{i j} \frac{\varepsilon\left(K_{i}\right)-\varepsilon\left(\bar{K}_{i}\right)}{q_{i}-q_{i}^{-1}} ;
\end{aligned}
$$

If $E_{i}$ is type 1 , then

$$
\begin{aligned}
\Delta\left(K_{j}\right) \Delta\left(E_{i}\right) & =\left(K_{j} \otimes K_{j}\right)\left(1 \otimes E_{i}+E_{i} \otimes K_{i}\right) \\
& =K_{j} \otimes K_{j} E_{i}+K_{j} E_{i} \otimes K_{j} K_{i} \\
& =q_{i}^{a_{i j}} K_{j} \otimes E_{i} K_{j}+q_{i}^{a_{i j}} E_{i} K_{j} \otimes K_{i} K_{j} \\
& =q_{i}^{a_{i j}} \Delta\left(E_{i}\right) \Delta\left(K_{j}\right)
\end{aligned}
$$

if $E_{i}$ is type 2 , then

$$
\begin{aligned}
\Delta\left(K_{j}\right) \Delta\left(E_{i}\right) \Delta\left(\bar{K}_{j}\right) & =\left(K_{j} \otimes K_{j}\right)\left(J \otimes E_{i}+E_{i} \otimes K_{i}\right)\left(\bar{K}_{j} \otimes \bar{K}_{j}\right) \\
& =K_{j} \bar{K}_{j} \otimes K_{j} E_{i} \bar{K}_{j}+K_{j} E_{i} \bar{K}_{j} \otimes K_{j} K_{i} \bar{K}_{j} \\
& =q_{i}^{a_{i j}} J \otimes E_{i}+q_{i}^{a_{i j}} E_{i} \otimes K_{i} \\
& =q_{i}^{a_{i j}} \Delta\left(E_{i}\right) .
\end{aligned}
$$

Therefore, $\Delta$ keeps the relation (2.11) for $E_{i}$ 's. The similar argument can show that $\Delta$ also keeps the relation $(2.11)$ for $F_{i}$ 's.

Now we examine the identity

$$
\Delta\left(E_{i}\right) \Delta\left(F_{j}\right)-\Delta\left(F_{j}\right) \Delta\left(E_{i}\right)=\delta_{i j} \frac{\Delta\left(K_{i}\right)-\Delta\left(\bar{K}_{i}\right)}{q_{i}-q_{i}^{-1}} .
$$

The following cases should be considered:

1. $E_{i}$ is type 1 and $F_{i}$ is type 1 ,

2. $E_{i}$ is type 1 and $F_{i}$ is type 2 ,

3. $E_{i}$ is type 2 and $F_{i}$ is type 1 ,

4. $E_{i}$ is type 2 and $F_{i}$ is type 2 . 
For the case 2, it is noted that

$$
E_{i} \bar{K}_{j}=q_{i}^{a_{i j}} \bar{K}_{j} E_{i}=q^{d_{i} a_{i j}} \bar{K}_{j} E_{i}=q^{\left(\alpha_{i}, \alpha_{j}\right)} \bar{K}_{j} E_{i}
$$

and

$$
K_{i} F_{j}=K_{i} F_{j} J=K_{i} F_{j} \bar{K}_{j} K_{i}=q_{j}^{-a_{j i}} F_{j} K_{i}=q^{-d_{j} a_{j i}} F_{j} K_{i}=q^{-\left(\alpha_{i}, \alpha_{j}\right)} F_{j} K_{i} .
$$

The later identity holds since $K_{i} J=K_{i}$ and $J$ is central in $\mathfrak{w}_{q}^{\mathrm{d}}(\mathfrak{g})$. Then, it is easy to see that

$$
\begin{aligned}
\Delta\left(E_{i}\right) \Delta\left(F_{j}\right)-\Delta\left(F_{j}\right) \Delta\left(E_{i}\right) & =\bar{K}_{j} \otimes\left(E_{i} F_{j}-F_{j} E_{i}\right)+\left(E_{i} F_{j}-F_{j} E_{i}\right) \otimes K_{i} \\
& =\delta_{i j} \bar{K}_{j} \otimes \frac{K_{i}-\bar{K}_{i}}{q_{i}-q_{i}^{-1}}+\delta_{i j} \frac{K_{i}-\bar{K}_{i}}{q_{i}-q_{i}^{-1}} \otimes K_{i} \\
& =\delta_{i j} \frac{K_{i} \otimes K_{i}-\bar{K}_{i} \otimes \bar{K}_{i}}{q_{i}-q_{i}^{-1}}=\delta_{i j} \frac{\Delta\left(K_{i}\right)-\Delta\left(\bar{K}_{i}\right)}{q_{i}-q_{i}^{-1}} .
\end{aligned}
$$

We have shown that $\Delta$ keeps the relation (2.12) for the case 2. For the other cases the proof is similar. To see the map $\Delta$ keeps the quantum Serre relations (2.13) and (2.14), we should consider several cases according to the type of $\left\{E_{i}, E_{j}\right\}$ or $\left\{F_{i}, F_{j}\right\}(i \neq j)$. In fact, for each case, the argument is more or less the same as the case of $U_{q}(\mathfrak{g})$ (see [10, p.67-68]).

Therefore, $\Delta$ and $\varepsilon$ can be extended to algebra morphisms from $\mathfrak{w}_{q}^{\mathrm{d}}(\mathfrak{g})$ to $\mathfrak{w}_{q}^{\mathrm{d}}(\mathfrak{g}) \otimes \mathfrak{w}_{q}^{\mathrm{d}}(\mathfrak{g})$ and from $\mathfrak{w}_{q}^{\mathrm{d}}(\mathfrak{g})$ to $k$ respectively.

By the above relations it can be shown that

$$
\begin{aligned}
(\Delta \otimes \mathrm{id}) \Delta(X) & =(\mathrm{id} \otimes \Delta) \Delta(X), \\
(\varepsilon \otimes \mathrm{id}) \Delta(X) & =(\mathrm{id} \otimes \varepsilon) \Delta(X)=X
\end{aligned}
$$

for any $X=E_{i}, F_{i}, K_{i}$ or $\bar{K}_{i}$. Let $\mu$ and $\eta$ be the product and the unit of $\mathfrak{w}_{q}^{\text {d }}(\mathfrak{g})$ respectively, then $\left(\mathfrak{w}_{q}^{\mathrm{d}}(\mathfrak{g}), \mu, \eta, \Delta, \varepsilon\right)$ becomes into a bialgebra.

It is easy to see that

$$
\begin{aligned}
T\left(\bar{K}_{i}\right) T\left(K_{j}\right) & =T\left(K_{j}\right) T\left(\bar{K}_{i}\right), \\
T(J) T\left(K_{i}\right) & =T\left(K_{i}\right), \\
T(J) T\left(\bar{K}_{i}\right) & =T\left(\bar{K}_{i}\right), \\
T\left(F_{j}\right) T\left(E_{i}\right)-T\left(E_{i}\right) T\left(F_{j}\right) & =\delta_{i j} \frac{T\left(K_{i}\right)-T\left(\bar{K}_{i}\right)}{q_{i}-q_{i}^{-1}},
\end{aligned}
$$

and $E_{i}$ is either type 1 or type 2, the map $T$ keeps the anti-relation of (2.11). The argument for $F_{i}$ is similar. Moreover, $T$ also keeps the anti-relation for quantum Serre relations. For example, for $1 \leq i, j \leq n$ with $i \neq j$, let $r=1-a_{i j}$, we have

$$
\begin{aligned}
& \sum_{s=0}^{r}(-1)^{s}\left[\begin{array}{l}
r \\
s
\end{array}\right]_{q_{i}} T\left(E_{i}\right)^{s} T\left(E_{j}\right) T\left(E_{i}\right)^{r-s} \\
& =\sum_{s=0}^{r}(-1)^{s}\left[\begin{array}{l}
r \\
s
\end{array}\right]_{q_{i}}(-1)^{r+1}\left(E_{i} \bar{K}_{i}\right)^{s}\left(E_{j} \bar{K}_{j}\right)\left(E_{i} \bar{K}_{i}\right)^{r-s} \\
& =-q_{j}^{2} q_{i}^{r^{2}+r+r a_{i j}} \bar{K}_{i}^{r} \bar{K}_{j} \sum_{s=0}^{r}(-1)^{r-s}\left[\begin{array}{c}
r \\
s
\end{array}\right]_{q_{i}} E_{i}^{s} E_{j} E_{i}^{r-s}=0 .
\end{aligned}
$$


Here we use the formula (2.16) and (2.17). The argument for $F_{i}$ is similar. Therefore, $T$ can be extended to an anti-algebra morphism from $\mathfrak{w}_{q}^{\mathrm{d}}(\mathfrak{g})$ to $\mathfrak{w}_{q}^{\mathrm{d}}(\mathfrak{g})$ respectively.

Recall that the convolution product in the bialgebra $\left(\mathfrak{w}_{q}^{\mathrm{d}}(\mathfrak{g}), \mu, \eta, \Delta, \varepsilon\right)$ is defined in the similar way to the standard one(see e.g. [9]) as

$$
(f * g)(X)=\mu(f \otimes g) \Delta(X)
$$

for all $f, g \in \operatorname{Hom}\left(\mathfrak{w}_{q}^{\mathrm{d}}(\mathfrak{g}), \mathfrak{w}_{q}^{\mathrm{d}}(\mathfrak{g})\right)$ and $X \in \mathfrak{w}_{q}^{\mathrm{d}}(\mathfrak{g})$. It is noted that if $E_{i}$ is type 2 , then

$$
J E_{i}=K_{i} \bar{K}_{i} E_{i}=q_{i}^{-2} K_{i} \bar{K}_{i} K_{i} E_{i} \bar{K}_{i}=q_{i}^{-2} K_{i} E_{i} \bar{K}_{i}=E_{i} .
$$

The same argument shows that $F_{i} J=F_{i} J=F_{i}$ if $F_{i}$ is type 2. Let id denote identity map in $\operatorname{hom}_{k}\left(\mathfrak{w}_{q}^{\mathrm{d}}(\mathfrak{g}), \mathfrak{w}_{q}^{\mathrm{d}}(\mathfrak{g})\right)$.

Lemma 3.3 Let $X$ be $E_{i}, F_{i}, K_{i}$, or $\bar{K}_{i}$, then

$$
\begin{aligned}
& (i d * T * i d)(X)=i d(X), \\
& (T * i d * T)(X)=T(X) .
\end{aligned}
$$

Proof. It is easy for $X=K_{i}, \bar{K}_{i}$. We consider $X=E_{i}$, as an example. We set

$$
\Delta_{2}=(\mathrm{id} \otimes \Delta) \circ \Delta \text {. }
$$

If $E_{i}$ is type 1 , then

$$
\Delta_{2}\left(E_{i}\right)=1 \otimes 1 \otimes E_{i}+1 \otimes E_{i} \otimes K_{i}+E_{i} \otimes K_{i} \otimes K_{i} .
$$

It follows that

$$
\begin{aligned}
(\mathrm{id} * T * \mathrm{id})\left(E_{i}\right) & =T(1) E_{i}+T\left(E_{i}\right) K_{i}+E_{i} T\left(K_{i}\right) K_{i} \\
& =E_{i}-E_{i} \bar{K}_{i} K_{i}+E_{i} \bar{K}_{i} K_{i}=\mathrm{id}\left(E_{i}\right)
\end{aligned}
$$

and

$$
\begin{aligned}
(T * \mathrm{id} * T)\left(E_{i}\right) & =T(1) T\left(E_{i}\right)+T(1) E_{i} T\left(K_{i}\right)+T\left(E_{i}\right) K_{i} T\left(K_{i}\right) \\
& =-E_{i} \bar{K}_{i}+E_{i} \bar{K}_{i}-E_{i} \bar{K}_{i} K_{i} \bar{K}_{i} \\
& =-E_{i} \bar{K}_{i}=T\left(E_{i}\right) .
\end{aligned}
$$

If $E_{i}$ is type 2 , then

$$
\Delta_{2}\left(E_{i}\right)=J \otimes J \otimes E_{i}+J \otimes E_{i} \otimes K_{i}+E_{i} \otimes K_{i} \otimes K_{i} .
$$

It also deduce that

$$
\begin{aligned}
(\mathrm{id} * T * \mathrm{id})\left(E_{i}\right) & =J T(J) E_{i}+J T\left(E_{i}\right) K_{i}+E_{i} T\left(K_{i}\right) K_{i} \\
& =J E_{i}-E_{i} \bar{K}_{i} K_{i}+E_{i} \bar{K}_{i} K_{i}=J E_{i}=\mathrm{id}\left(E_{i}\right)
\end{aligned}
$$

since $J E_{i}=E_{i}$, and

$$
\begin{aligned}
(T * \mathrm{id} * T)\left(E_{i}\right) & =T(J) J T\left(E_{i}\right)+T(J) E_{i} T\left(K_{i}\right)+T\left(E_{i}\right) K_{i} T\left(K_{i}\right) \\
& =-E_{i} \bar{K}_{i}+E_{i} \bar{K}_{i}-E_{i} \bar{K}_{i} K_{i} \bar{K}_{i} \\
& =-E_{i} \bar{K}_{i}=T\left(E_{i}\right)
\end{aligned}
$$


As for $F_{i}$, the argument is similar. The proof of the lemma is finished.

In order to conclude that the antipode axioms hold on arbitrary elements, the following two facts are to be used.

(a) The co-products of the generators are bilinear expressions of generators;

(b) one of $(\mathrm{id} * T)(X)$ and $T * \operatorname{id}(X)$ is a central element of $\mathfrak{w}_{q}^{\mathrm{d}}(\mathfrak{g})$ for $X$ being the generators $K_{i}, \bar{K}_{i}, E_{i}, F_{i}$.

The fact (a) is obvious. To see (b), we note the fact that (id $* T)(X)=\varepsilon(X) J$ for $X=K_{i}, \bar{K}_{i}, E_{i}(1 \leq i \leq n)$ and $F_{i}$ of type 2 . Hence $(\mathrm{id} * T)(X)$ is in the center of $\mathfrak{w}_{q}^{\mathrm{d}}(\mathfrak{g})$. However, if $X=F_{i}$ is type 1 , the $(\mathrm{id} * T)(X)=(1-J) F_{i}$ may not be a central element, but $(T *$ id $)\left(F_{i}\right)=\varepsilon\left(F_{i}\right) J$ is in the center. Similarly, $(T *$ id $)(X)$ for $X=K_{i}, \bar{K}_{i}, F_{i}$ $(1 \leq i \leq n)$ and $E_{i}$ is of type 2 , in the center of $\mathfrak{w}_{q}^{\mathrm{d}}(\mathfrak{g})$. However, if $X=E_{i}$ is type 1 , the $(T * \mathrm{id})(X)=(1-J) E_{i}$ may not be a central element, but $(\mathrm{id} * T)\left(E_{i}\right)$ is in the center of $\mathfrak{w}_{q}^{\mathrm{d}}(\mathfrak{g})$. This means that (b) holds.

It is noted that $E_{i}(1-J) F_{j}=F_{j}(1-J) E_{i}$ for all $i, j \in\{1,2, \cdots, n\}$ by the relation (2.12). Therefore, if $E_{i}$ ( resp. $\left.F_{i}\right)$ is type $1,\left(T *\right.$ id) $\left(E_{i}\right)$ (resp. (id $\left.* T\right)\left(F_{i}\right)$ ) commutates with all $F_{j}$ ( resp. all $E_{j}$ ), and $K_{j}, \bar{K}_{j}(1 \leq j \leq n)$.

We should show the claim that if

$$
\begin{aligned}
& (\text { id } * T * \mathrm{id})(x)=x,(T * \mathrm{id} * T)(x)=T(x) ; \\
& (\text { id } * T * \mathrm{id})(y)=y, \quad(T * \mathrm{id} * T)(y)=T(y),
\end{aligned}
$$

for all $x$ and $y$ being generators $E_{i}, F_{i}, K_{i}, \bar{K}_{i}$, then

$$
(\mathrm{id} * T * \mathrm{id})(x y)=x y,(T * \mathrm{id} * T)(x y)=T(x y) .
$$

However, it is considerable direct by the above facts. Now, that the antipode axioms hold on arbitrary elements is obvious by induction.

If we assume that with the operations $\mu, \eta, \Delta, \varepsilon$ the algebra $\mathfrak{w}_{q}^{\mathrm{d}}(\mathfrak{g})$ would possess an antipode $S$ so as to become a Hopf algebra, then $S$ should satisfy $(S * \mathrm{id})(J)=\eta \varepsilon(J)$, and it would follow that $S(J) J=1$ and $J$ is invertible. It is impossible since $J(1-J)=0$. This implies that $\mathfrak{w}_{q}^{\mathrm{d}}(\mathfrak{g})$ is not a Hopf algebra with the above operators. The proof of Theorem 3.1 is finished.

It should be noted that if $\mathfrak{g}=\mathfrak{s l}_{n}$, the algebra $\mathfrak{w}_{q}^{\mathrm{d}}(\mathfrak{g})$ is just the mixtures $\mathfrak{w} \mathfrak{s l}_{q}^{d}(n)$ in [2]. In particular, if $\mathfrak{g}=\mathfrak{s l}_{2}, \mathfrak{w}_{q}^{\mathrm{d}}(\mathfrak{g})$ where $\mathbf{d}=(1 \mid 1)$ (resp. $\mathbf{d}=(0 \mid 0)$ ) coincides with $\mathfrak{w s l}_{q}(2)$ $\left(\right.$ resp. $\left.v \mathfrak{s l}_{q}(2)\right)$ given in [12].

\section{The basis of $\mathfrak{w}_{q}^{\mathbf{d}}(\mathfrak{g})$}

One can find the relationship between $U_{q}(\mathfrak{g})$ and the quantum algebra $\mathfrak{w}_{q}^{\mathrm{d}}(\mathfrak{g})$ as follows.

Proposition $4.1 \mathfrak{w}_{q}^{d}(\mathfrak{g}) /\langle J-1\rangle \cong U_{q}(\mathfrak{g})$.

Proof. It is obvious by cancelling $K_{i}$. 
In fact, we can give a more explicit relationship between $\mathfrak{w}_{q}^{\mathrm{d}}(\mathfrak{g})$ and $U_{q}(\mathfrak{g})$. For this purpose, we let $w_{q}=\mathfrak{w}_{q}^{\mathrm{d}}(\mathfrak{g}) J$ and $\bar{w}_{q}=\mathfrak{w}_{q}^{\mathrm{d}}(\mathfrak{g})(1-J)$. We have the following decomposition.

Proposition 4.2 As algebras $\mathfrak{w}_{q}^{d}(\mathfrak{g})=w_{q} \oplus \bar{w}_{q}$. Moreover, $w_{q} \cong U_{q}(\mathfrak{g})$ as Hopf algebras.

Proof. Noting that $J$ is a central idempotent, we see that $w_{q}$ and $\bar{w}_{q}$ are ideals of $\mathfrak{w}_{q}^{\mathrm{d}}(\mathfrak{g})$. It follows that

$$
\mathfrak{w}_{q}^{\mathrm{d}}(\mathfrak{g})=w_{q} \oplus \bar{w}_{q}
$$

as algebras. Moreover, it is easy to see that $w_{q}$ is generated by $E_{i} J, F_{i} J, K_{i}, \bar{K}_{i}$ and $J$ subject to the relations (2.8)-(2.10) and

$$
\begin{aligned}
& K_{i}\left(E_{j} J\right)=q_{i}^{a_{i j}}\left(E_{j} J\right) K_{i}, \quad \bar{K}_{i}\left(E_{j} J\right)=q_{i}^{-a_{i j}}\left(E_{j} J\right) \bar{K}_{i}, \\
& K_{i}\left(F_{j} J\right)=q_{i}^{-a_{i j}}\left(F_{j} J\right) K_{i}, \quad \bar{K}_{i}\left(F_{j} J\right)=q_{i}^{a_{i j}}\left(F_{j} J\right) \bar{K}_{i}, \\
& \left(E_{i} J\right)\left(F_{j} J\right)-\left(F_{j} J\right)\left(E_{i} J\right)=\delta_{i j} \frac{K_{i}-\bar{K}_{i}}{q_{i}-q_{i}^{-1}} ; \\
& \sum_{s=0}^{1-a_{i j}}(-1)^{s}\left[\begin{array}{c}
1-a_{i j} \\
s
\end{array}\right]_{q_{i}}\left(E_{i} J\right)^{1-a_{i j}-s}\left(E_{j} J\right)\left(E_{i} J\right)^{s}=0, \text { if } i \neq j ; \\
& \sum_{s=0}^{1-a_{i j}}(-1)^{s}\left[\begin{array}{c}
1-a_{i j} \\
s
\end{array}\right]_{q_{i}}\left(F_{i} J\right)^{1-a_{i j}-s}\left(F_{j} J\right)\left(F_{i} J\right)^{s}=0, \text { if } i \neq j .
\end{aligned}
$$

Here $J$ can be viewed as the identity of $w_{q}$. At this point of view $w_{q}$ becomes a Hopf algebra, in which the co-multiplication $\Delta$ is

$$
\begin{aligned}
& \Delta\left(E_{i} J\right)=J \otimes E_{i} J+E_{i} J \otimes K_{i}, \\
& \Delta\left(F_{i} J\right)=F_{i} J \otimes J+\bar{K}_{i} \otimes F_{i} J, \\
& \Delta\left(K_{i}\right)=K_{i} \otimes K_{i}, \Delta\left(\bar{K}_{i}\right)=\bar{K}_{i} \otimes \bar{K}_{i} .
\end{aligned}
$$

The counit $\varepsilon$ is

$$
\varepsilon\left(E_{i} J\right)=\varepsilon\left(F_{i} J\right)=0, \varepsilon\left(K_{i}\right)=\varepsilon\left(\bar{K}_{i}\right)=1 .
$$

The antipode $S$ is

$$
S\left(E_{i} J\right)=-\left(E_{i} J\right) \bar{K}_{i}, S\left(F_{i} J\right)=-K_{i}\left(F_{i} J\right), S\left(K_{i}\right)=\bar{K}_{i}, S\left(\bar{K}_{i}\right)=K_{i} .
$$

Let $\rho$ be the algebra morphism from $U_{q}(\mathfrak{g})$ to $w_{q}$ defined by

$$
\rho\left(e_{i}\right)=E_{i} J, \rho\left(f_{i}\right)=F_{i} J, \rho\left(k_{i}\right)=K_{i}, \rho\left(k_{i}^{-1}\right)=\bar{K}_{i} .
$$

It is straightforward to see that $\rho$ is a Hopf algebra isomorphism.

For the ideal $\bar{w}_{q}$ of $\mathfrak{w}_{q}^{\mathrm{d}}(\mathfrak{g})$, some conventions should be noted. Let

$$
\mathrm{d}=\left(\kappa_{1}, \cdots, \kappa_{n} \mid \bar{\kappa}_{1}, \cdots, \bar{\kappa}_{n}\right)
$$

be a binary sequence. If $\kappa_{i}$ (resp. $\left.\bar{\kappa}_{i}\right), 1 \leq i \leq n$ is zero, and hence that $E_{i}$ (resp. $F_{i}$ ) is type 2 , then $E_{i}(1-J)=0$ (resp. $F_{i}(1-J)=0$ ); if $\kappa_{i}$ (resp. $\left.\bar{\kappa}_{i}\right), 1 \leq i \leq n$ is non-zero, and hence that $E_{i}\left(\operatorname{resp} . F_{i}\right)$ is type 1 , then $E_{i}(1-J) \neq 0\left(\right.$ resp. $\left.F_{i}(1-J) \neq 0\right)$. Let

$$
\mathfrak{d}=\left\{i \mid \kappa_{i} \neq 0\right\} \text { and } \overline{\mathfrak{d}}=\left\{i \mid \bar{\kappa}_{i} \neq 0\right\}
$$


and

$$
X_{i}=E_{i}(1-J), Y_{j}=F_{j}(1-J),
$$

where $i \in \mathfrak{d}, j \in \overline{\mathfrak{d}}$. It is easy to see that $\left\{X_{i}, Y_{j} \mid i \in \mathfrak{d}, j \in \overline{\mathfrak{d}}\right\} \cup\{1-J\}$ generate the ideal $\bar{w}_{q}$ with enjoying the first relation

$$
X_{i} Y_{j}=Y_{j} X_{i}, \quad \text { for all } i \in \mathfrak{d}, j \in \overline{\mathfrak{d}}
$$

from the relation (2.12).

To see what other relations $X_{i}$ and $Y_{i}$ enjoy, we consider the following two extreme cases

1. the case

$$
\overline{\mathrm{d}}:=(\underbrace{1, \cdots, 1}_{n \text { copies }} \underbrace{1, \cdots, 1}_{n \text { copies }}) .
$$

In this case, $\mathfrak{d}=\{1, \cdots, n\}$ and $\overline{\mathfrak{d}}=\{1, \cdots, n\}$. From the quantum Serre relations (2.13) and (2.14), we get that

$$
\begin{aligned}
& \sum_{s=0}^{1-a_{i j}}(-1)^{s}\left[\begin{array}{c}
1-a_{i j} \\
s
\end{array}\right]_{q_{i}}\left(X_{i}\right)^{1-a_{i j}-s} X_{j}\left(X_{i}\right)^{s}=0, \text { if } i \neq j, \\
& \sum_{s=0}^{1-a_{i j}}(-1)^{s}\left[\begin{array}{c}
1-a_{i j} \\
s
\end{array}\right]_{q_{i}}\left(Y_{i}\right)^{1-a_{i j}-s} Y_{j}\left(Y_{i}\right)^{s}=0, \text { if } i \neq j,
\end{aligned}
$$

and other relations corresponding to (2.8)-(2.11) would be vanished automatically. This means that the ideal $\bar{w}_{q}$ can be understood as an algebra generated by $X_{i}, Y_{i}, 1 \leq i \leq n$. with an identity $1-J$ subject to the relations (4.6)-(4.8).

2. the case

$$
\underline{\mathrm{d}}:=(\underbrace{0, \cdots, 0}_{n \text { copies }} \underbrace{0, \cdots, 0}_{n \text { copies }}) .
$$

In this case, $\mathfrak{d}$ and $\overline{\mathfrak{d}}$ are empty and $\bar{w}_{q}=k(1-J)$.

In general, three cases should be considered.

1. $\mathfrak{d} \neq \emptyset$ and $\overline{\mathfrak{d}}=\emptyset$;

2. $\mathfrak{d}=\emptyset$ and $\overline{\mathfrak{d}} \neq \emptyset$;

3. $\mathfrak{d} \neq \emptyset$ and $\overline{\mathfrak{d}} \neq \emptyset$.

In the first case, as an algebra $\bar{w}_{q}$ is generated by $X_{i}, i \in \mathfrak{d}$ with an identity $1-J$ subject to the relations (4.7) with $i, j \in \mathfrak{d}$. In the second case, as an algebra $\bar{w}_{q}$ is generated by $Y_{i}, i \in \overline{\mathfrak{d}}$ with an identity $1-J$ subject to the relations (4.8) with $i, j \in \overline{\mathfrak{d}}$. In the third case, $\bar{w}_{q}$ can be viewed as an algebra generated by $X_{i}, Y_{j}, i \in \mathfrak{d}, j \in \overline{\mathfrak{d}}$ with an identity $1-J$ subject to the relations (4.6)-(4.8).

To consider the PBW basis of $\mathfrak{w}_{q}^{\mathrm{d}}(\mathfrak{g})$, we need some knowledge of braid groups. We define a simple reflection $s_{i}$ by

$$
s_{i}\left(\alpha_{j}\right)=\alpha_{j}-a_{i j} \alpha_{i}
$$


for all $i$ and $j$. Let $W$ be the Weyl group of $R$; it is the subgroup of $\operatorname{GL}\left(\mathbb{Z}^{n}\right)$ generated by the refelctions $s_{i}(1 \leq i \leq n)$. Let $\ell(w)$ be the usual length function on $W$ with respect to the generators $\left\{s_{1}, \cdots, s_{n}\right\}$. Let $R^{+}$be the set of positive roots of $R$ with respect to the set of simple roots $\Pi$ and $\ell_{0}=\left|R^{+}\right|$. For each pair $1 \leq i, j \leq n$ with $i \neq j$, we let $r=-a_{i j}$.

As is known in [10], for each $1 \leq i \leq n$, there is a unique automorphism $T_{i}: U_{q}(\mathfrak{g}) \rightarrow$ $U_{q}(\mathfrak{g})$ such that

$$
\begin{aligned}
& T_{i}\left(e_{i}\right)=-f_{i} k_{i}, T_{i}\left(f_{i}\right)=-k_{i}^{-1} e_{i}, \\
& T_{i}\left(k_{\mu}\right)=k_{s_{i}(\mu)}, \\
& T_{i}\left(e_{j}\right)=\sum_{k=0}^{r}(-1)^{i} q_{i}^{-k} e_{i}^{(r-i)} e_{j} e_{i}^{(i)}, \\
& T_{i}\left(f_{j}\right)=\sum_{k=0}^{r}(-1)^{i} q_{i}^{k} f_{i}^{(i)} f_{j} f_{i}^{(r-i)} .
\end{aligned}
$$

They are called Lusztig's symmetries. It is well known that $\left\{T_{i} \mid 1 \leq i \leq n\right\}$ satisfies the braid relations, that is

$$
\begin{aligned}
& T_{i} T_{j} T_{i}=T_{j} T_{i} T_{j}, \quad \text { if } s_{i} s_{j} \text { of order } 2, \\
& T_{i} T_{j} T_{i} T_{j}=T_{j} T_{i} T_{j} T_{i}, \quad \text { if } s_{i} s_{j} \text { of order } 4, \\
& T_{i} T_{j} T_{i} T_{j} T_{i} T_{j}=T_{j} T_{i} T_{j} T_{i} T_{j} T_{i}, \quad \text { if } s_{i} s_{j} \text { of order } 6 .
\end{aligned}
$$

Therefore, the above facts allow us to define for each $w \in W$ an automorphism $T_{w}$ of $U_{q}(\mathfrak{g})$ as follows. For $w=1$ set $T_{1}=1$ (the identity). For $w \neq 1$ choose a reduced expression $w=s_{i_{1}} \cdots s_{i_{m}}$ and set

$$
T_{w}=T_{i_{1}} \cdots T_{i_{m}}
$$

It is independent of the reduced expression. Let $U_{q}^{+}$(resp. $U_{q}^{-}$and $U_{q}^{0}$ ) be the subalgebra of $U_{q}(\mathfrak{g})$ generated by $e_{i}$ (resp. $f_{i}$ and $\left.k_{i}, k_{i}^{-1}\right), 1 \leq i \leq n$. Let $w_{0}$ be the longest element in $W$ and let $w_{0}=s_{i_{1}} \cdots s_{i_{t}}$ be a reduced expression. Let $\mathbb{N}$ be the set of non-negative numbers. According to this order we denote $a=\left(a_{t}, \cdots, a_{1}\right) \in \mathbb{N}^{\ell_{0}}$ and

$$
e^{a}=T_{i_{1}} \cdots T_{i_{t-1}}\left(e_{i_{t}}^{a_{t}}\right) \cdots T_{i_{1}} T_{i_{2}}\left(e_{i_{3}}^{a_{3}}\right) T_{i_{1}}\left(e_{i_{2}}^{a_{2}}\right) e_{i_{1}}^{a_{1}} .
$$

The following theorem is well known.

Theorem 4.3 (cf. [10, Theorem 8.24]) The elements $e^{a}$ (resp. $f^{a}$ ) for $a \in \mathbb{N}^{\ell_{0}}$, are linearly independent and a basis of $U_{q}^{+}$(resp. $\left.U_{q}^{-}\right)$.

We note that the multiplication map

$$
U_{q}^{-} \otimes U_{q}^{0} \otimes U_{q}^{+} \rightarrow U_{q}(\mathfrak{g}), \quad u_{1} \otimes u_{2} \otimes u_{3} \rightarrow u_{1} u_{2} u_{3}
$$

is an isomorphism of vector spaces.

For $s=\left(s_{1}, s_{2}, \cdots, s_{n}\right) \in \mathbb{Z}^{n}$, we define

$$
P^{s}=P_{1}^{s_{1}} P_{2}^{s_{2}} \cdots P_{n}^{s_{n}} .
$$

First, let us examine two examples. 
Example 4.4 The set

$$
\left\{F^{b} P^{s} E^{a} J \mid a, b \in \mathbb{N}^{\ell_{0}}, s \in \mathbb{Z}^{n}\right\} \bigcup\left\{F^{b} E^{a}(1-J) \mid a, b \in \mathbb{N}^{\ell_{0}}\right\}
$$

forms a basis of $\mathfrak{w}_{q}^{\bar{d}}(\mathfrak{g})$.

Proof. Let $w_{q}^{0}$ be the subalgebra generated by $\left\{K_{i}, \bar{K}_{i} \mid 1 \leq i \leq n\right\}$. It is easy to see that $P^{s}(s \in \mathbb{Z})$ forms a basis of $w_{q}^{0}$.

Let $w_{q}^{+}\left(\right.$resp. $\left.w_{q}^{-}\right)$denote the subalgebra generated by $E_{i} J\left(\operatorname{resp} . F_{i} J\right), 1 \leq i \leq n$.

We replace $e_{i_{k}}$ where $k=1, \cdots, t$ in the right hand side of (4.9) by $E_{i_{k}} J$ (resp. $\left.E_{i_{k}}(1-J)\right)$, and the corresponding left hand side by $(E J)^{a}\left(\operatorname{resp} .(E(1-J))^{a}\right)$. By Theorem 4.3 the set $\left\{(E J)^{a} \mid a \in \mathbb{N}^{\ell_{0}}\right\}$ (resp. $\left\{(F J)^{b} \mid b \in \mathbb{N}^{\ell_{0}}\right\}$ ) forms a basis of $w_{q}^{+}$ (resp. $w_{q}^{-}$). It is easy to see that

$$
(F J)^{b} P^{s}(E J)^{a}=F^{b} P^{s} E^{a} J .
$$

It follows from (4.10) that

$$
\left\{F^{a} P^{s} E^{b} J \mid a, b \in \mathbb{N}^{\ell_{0}}, s \in \mathbb{Z}^{n}\right\}
$$

forms a basis of $w_{q}$.

Similarly, $\left\{F^{a} E^{b}(1-J) \mid a, b \in \mathbb{N}^{\ell_{0}}\right\}$ forms a basis of $\bar{w}_{q}$. The proof is completed by Proposition 4.2.

In a similar way we can get that

Example 4.5 The set

$$
\left\{F^{b} P^{s} E^{a} J \mid a, b \in \mathbb{N}^{\ell_{0}}, s \in \mathbb{Z}^{n}\right\} \bigcup\{1-J\}
$$

forms a basis of $\mathfrak{w} \frac{d}{q}(\mathfrak{g})$.

In general, let both $i$ and $j$ be in $\mathfrak{d}$ or in $\overline{\mathfrak{d}}$, we say that $i \sim j$ if there exist some sequence $i=\gamma_{1}, \cdots, \gamma_{p}=j$ in $\mathfrak{d}$, where $\gamma_{p}$ is in $\mathfrak{d}$ or $\overline{\mathfrak{d}}, p$ is some positive integer, such that $\left(\alpha_{\gamma_{i}}, \alpha_{\gamma_{i+1}}\right) \neq 0$ for all $i=1, \cdots, p-1$. This is an equivalent relation. Let $\mathfrak{s}$ and $\overline{\mathfrak{s}}$ be the set of equivalent classes on $\mathfrak{d}$ and $\overline{\mathfrak{d}}$ respectively. If $\mathfrak{e}$ is an element in $\mathfrak{s}$ or $\overline{\mathfrak{s}}$, it is obvious that $C_{\mathfrak{e}}=\left(a_{i j}\right)_{i, j \in \mathfrak{e}}$ is also a symmetrizable Cartan matrix. If $\mathfrak{e}_{1} \neq \mathfrak{e}_{2}$ in $\mathfrak{s}$ or $\overline{\mathfrak{s}}$, and $i \in \mathfrak{e}_{1}$ and $j \in \mathfrak{e}_{2}$, then $E_{i} E_{j}=E_{j} E_{i}$ and $F_{i} F_{j}=F_{j} F_{i}$, and hence $X_{i} X_{j}=X_{j} X_{i}$ and $Y_{i} Y_{j}=Y_{j} Y_{i}$ respectively. Let $W_{\mathfrak{e}}$ be the Weyl group corresponding to the equivalent class $\mathfrak{e}$ on $\mathfrak{d}$ or $\overline{\mathfrak{d}}$. Let $w_{0}^{\mathfrak{e}}=s_{i_{1}} \cdots s_{i_{\mathfrak{e}}}$ be the longest element in $W_{\mathfrak{e}}$. Let $\ell_{\mathfrak{e}}=\left|w_{0}^{\mathfrak{e}}\right|=t_{\mathfrak{e}}$. We denote $a_{\mathfrak{e}}=\left(a_{t_{\mathfrak{e}}}, \cdots, a_{1}\right)$ according to this order and

$$
\begin{aligned}
X_{\mathfrak{e}}^{a_{\mathfrak{e}}} & =T_{i_{1}} \cdots T_{i_{t_{\mathfrak{e}}-1}}\left(E_{i_{t_{\mathfrak{e}}}}^{a_{t_{\mathfrak{e}}}}\right) \cdots T_{i_{1}} T_{i_{2}}\left(E_{i_{3}}^{a_{3}}\right) T_{i_{1}}\left(E_{i_{2}}^{a_{2}}\right) E_{i_{1}}^{a_{1}}(1-J), \\
Y_{\mathfrak{e}}^{a_{\mathfrak{e}}} & =T_{i_{1}} \cdots T_{i_{t_{\mathfrak{e}}-1}}\left(F_{i_{t_{\mathfrak{e}}}}^{a_{t_{\mathfrak{e}}}}\right) \cdots T_{i_{1}} T_{i_{2}}\left(F_{i_{3}}^{a_{3}}\right) T_{i_{1}}\left(F_{i_{2}}^{a_{2}}\right) F_{i_{1}}^{a_{1}}(1-J) .
\end{aligned}
$$

It is noted that

$$
X_{i} Y_{j}=Y_{j} X_{i}
$$


for all $i \in \mathfrak{d}, j \in \overline{\mathfrak{d}}$, and

$$
\mathfrak{d}=\bigcup_{\mathfrak{e} \in \mathfrak{s}} \mathfrak{e}, \quad \overline{\mathfrak{d}}=\bigcup_{\mathfrak{e} \in \overline{\mathfrak{s}}} \mathfrak{e}
$$

one sees that

$$
\left\{\prod_{\mathfrak{e} \in \mathfrak{s}} X_{\mathfrak{e}}^{a_{\mathfrak{e}}} \prod_{\overline{\mathfrak{e}} \in \overline{\mathfrak{s}}} Y_{\overline{\mathfrak{e}}}^{b_{\overline{\mathfrak{e}}}} \mid a_{\mathfrak{e}} \in \mathbb{N}^{\ell_{\mathfrak{e}}}, b_{\overline{\mathfrak{e}}} \in \mathbb{N}^{\ell_{\overline{\mathfrak{e}}}}\right\}
$$

forms a basis of $\bar{w}_{q}$. By Proposition 4.2 and the discussion above, it follows that

Theorem 4.6 The notations are kept as above. Then the set

$$
\left\{F^{b} P^{s} E^{a} J \mid a, b \in \mathbb{N}^{\ell_{0}}, s \in \mathbb{Z}^{n}\right\} \bigcup\left\{\prod_{\mathfrak{e} \in \mathfrak{s}} X_{\mathfrak{e}}^{a_{\mathfrak{e}}} \prod_{\overline{\mathfrak{e}} \in \overline{\mathfrak{s}}} Y_{\overline{\mathfrak{e}}}^{b_{\overline{\mathfrak{e}}}} \mid a_{\mathfrak{e}} \in \mathbb{N}^{\ell_{\mathfrak{e}}}, b_{\overline{\mathfrak{e}}} \in \mathbb{N}^{\ell_{\overline{\mathfrak{e}}}}\right\}
$$

forms a basis of $\mathfrak{w}_{q}^{d}(\mathfrak{g})$.

It is mentioned that the set

$$
\left\{E^{a} P^{s} F^{a} J \mid a, b \in \mathbb{N}^{\ell_{0}}, s \in \mathbb{Z}^{n}\right\} \bigcup\left\{\prod_{\mathfrak{e} \in \mathfrak{s}} X_{\mathfrak{e}}^{a_{\mathfrak{e}}} \prod_{\overline{\mathfrak{e}} \in \overline{\mathfrak{s}}} Y_{\overline{\mathfrak{e}}}^{b_{\overline{\mathfrak{c}}}} \mid a_{\mathfrak{e}} \in \mathbb{N}^{\ell_{\mathfrak{e}}}, b_{\overline{\mathfrak{e}}} \in \mathbb{N}^{\ell_{\overline{\mathfrak{e}}}}\right\}
$$

also forms a basis of $\mathfrak{w}_{q}^{\mathrm{d}}(\mathfrak{g})$.

Let us recall some basic facts used below. Let $C$ be a coalgebra. If the set $\left\{C_{n}\right\}_{n \geq 0}$ of subspaces of $C$ satisfies

1. $C_{n} \subseteq C_{n+1}$ and $C=\cup_{n \geq 0} C_{n}$

2. $\Delta\left(C_{n}\right) \subseteq \sum_{i=0}^{n} C_{i} \otimes C_{n-i}$,

then the set $\left\{C_{n}\right\}_{n \geq 0}$ is said to be a coalgebra filtration of $C$.

The following lemma is well known.

Lemma 4.7 ([14, Lemma 5.5.1]) Let $H$ be a bialgebra which contains subspaces $A_{0} \subset A_{1}$ such that

1. $A_{0}$ is a (unital) subalgebra of $H$ and $A_{1}$ is a left, and a right $A_{0}$-module;

2. $A_{1}$ generates $H$ as an algebra, and $1 \in A_{0}$;

3. $\Delta A_{0} \subseteq A_{0} \otimes A_{0}$ and $\Delta\left(A_{1}\right) \subseteq A_{1} \otimes A_{0}+A_{0} \otimes A_{1}$.

Then, if we set $A_{n}=\left(A_{1}\right)^{n}$ for all $n \geq 1,\left\{A_{n}\right\}$ is a coalgebra filtration of $H$ and $A_{0} \supseteq H_{0}$, where $H_{0}$ is the coradical of $H$.

The element $x \in \mathfrak{w}_{q}^{\mathrm{d}}(\mathfrak{g})$ is said to be a group-like element if $\Delta(x)=x \otimes x$. The set $G=G\left(\mathfrak{w}_{q}^{\mathrm{d}}(\mathfrak{g})\right)$ of all group-like elements of $\mathfrak{w}_{q}^{\mathrm{d}}(\mathfrak{g})$ can be determined.

A semigroup $S$ is called regular, if for every $x \in S$, there exists a $y \in S$ such that $x y x=x$ and $y x y=y$ and a monoid is a semigroup with identity. 
Proposition 4.8 The set of all group-like elements is $G=\left\{P^{s} \mid s \in \mathbb{Z}^{n}\right\} \bigcup\{1\}$, which forms a regular monoid under the multiplication of $\mathfrak{w}_{q}^{d}(\mathfrak{g})$.

Proof. Let $G$ be the setup as above. Let $A_{0}=k G$ and

$$
A_{1}=A_{0}\left(\sum_{i} k E_{i}+k F_{i}+A_{0}\right) A_{0} .
$$

It is easy to see that $A_{0} \subset A_{1}$ satisfies the hypotheses of Lemma 4.7 and $A_{0} \subseteq H_{0}$. Hence $H_{0}=A_{0}$. 4.8 .

It is mentioned that $\mathfrak{w}_{q}^{\mathrm{d}}(\mathfrak{g})$ is a pointed bialgebra with the coradical $k G$ by Proposition

\section{$5 \quad$ The automorphism group of $\mathfrak{w}_{q}^{\mathrm{d}}(\mathfrak{g})$}

If $(A, m, \mu, \Delta, \varepsilon, T)$ is a (weak) Hopf algebra, then a (weak) Hopf algebra automorphism $\varphi: A \rightarrow A$ is an invertible algebra homomorphism satisfying

$$
\begin{aligned}
& (\varphi \otimes \varphi) \circ \Delta=\Delta \circ \varphi, \\
& \varepsilon=\varepsilon \circ \varphi, \\
& \varphi \circ T=T \circ \varphi .
\end{aligned}
$$

The group of Hopf algebra automorphisms of $U_{q}(\mathfrak{g})$ was determined by several authors. See for example [4], [15]. Inspired by these considerations, we would like to determine the group of weak Hopf algebra automorphisms of $\mathfrak{w}_{q}^{\mathrm{d}}(\mathfrak{g})$ where $q$ is not a root of unity.

let $N=\left(k^{*}\right)^{n}$, and for $a=\left(a_{1}, \cdots, a_{n}\right) \in N$, we define a map $\phi_{a}: U_{q}(\mathfrak{g}) \rightarrow U_{q}(\mathfrak{g})$ by

$$
\phi_{a}\left(k_{i}\right)=k_{i}, \phi_{a}\left(e_{i}\right)=a_{i} e_{i}, \phi_{a}\left(f_{i}\right)=a_{i}^{-1} f_{i} .
$$

It is straightfoward to check that $\phi_{a}$ is a Hopf algebra automorphism of $U_{q}(\mathfrak{g})$. It is called $N$ the group of diagonal automorphisms of $U_{q}(\mathfrak{g})$.

Recall that the Dynkin diagram of $\mathfrak{g}$ is the weight graph $\Gamma$ with vertices $\mathfrak{n}=\{1,2, \cdots, n\}$ such that vertices $i$ and $j$ are connected by $a_{i j} a_{j i}$ edges, and vertex $i$ carries weight $d_{i}$. Let $\sigma$ be a automorphsim of Dynkin diagram $\Gamma$, that is $\sigma$ is a bijection of $\mathfrak{n}$ and

$$
d_{i} a_{i j}=d_{\sigma(i)} a_{\sigma(i) \sigma(j)}
$$

for all $1 \leq i, j \leq n$. If this is the case, there is an automorphism of Hopf algebra $U_{q}(\mathfrak{g})$, also denote by $\sigma$, given by

$$
\sigma\left(k_{i}\right)=k_{\sigma(i)}, \sigma\left(e_{i}\right)=e_{\sigma(i)}, \sigma\left(f_{i}\right)=f_{\sigma(i)} .
$$

We denote by $H$ the group of automorphisms of the Dynkin diagram. Also, $H$ acts on $N$ by the rules $\sigma \cdot a=\left(a_{\sigma(1)}, \cdots, a_{\sigma(n)}\right)$ and we have $\phi_{a} \sigma=\sigma \phi_{\sigma \cdot a}$. We will base on the following theorem to investigate the group of automorphisms of $\mathfrak{w}_{q}^{\mathrm{d}}(\mathfrak{g})$. 
Theorem 5.1 ([4, Corollary 4.3; 18, Theorem 2.1]) The group of Hopf algebra automorphism of $U_{q}(\mathfrak{g})$ is the semidirect product $N \rtimes H$ of the group of diagonal automorphism $N$ by the group of diagram automorphisms $H$.

Moreover, we also need some basic lemmas.

Lemma 5.2 If $x, y \in \bar{w}_{q}, a_{x}, b_{y} \neq 0$ and

$$
\begin{aligned}
& \Delta(x)=1 \otimes x+x \otimes K_{i}+a_{x}(1-J) \otimes E_{i} J \\
& \Delta(y)=\bar{K}_{i} \otimes y+y \otimes 1+b_{y} F_{i} J \otimes(1-J) .
\end{aligned}
$$

then $x=a_{x} E_{i}(1-J)$ and $y=b_{y} F_{i}(1-J)$.

Proof. The notations $\mathfrak{d}$ and $\overline{\mathfrak{d}}$ are as in Section 4 . Let $W_{\mathfrak{d}}$ be the Weyl group corresponding to $\mathfrak{d}$ and $w_{\mathfrak{d}}=s_{i_{1}} \cdots s_{i_{t_{\mathfrak{d}}}}$ be the longest element in $W_{\mathfrak{d}}$ and $\ell_{\mathfrak{d}}=t_{\mathfrak{d}}$. Similarly, we have the Weyl group $W_{\overline{\mathfrak{d}}}, w_{\overline{\mathfrak{d}}}=s_{j_{1}} \cdots s_{j_{\overline{\mathfrak{d}}-1}}, \ell_{\overline{\mathfrak{d}}}$ for the support set $\overline{\mathfrak{d}}$ in an obvious sense.

Let $\mathfrak{w}_{q}^{\mathrm{d}}(\mathfrak{g})^{+}$be the sub-bialgebra of $\mathfrak{w}_{q}^{\mathrm{d}}(\mathfrak{g})$ generated by $E_{i}, 1 \leq i \leq n$ and the set $G$ of group-likes of $\mathfrak{w}_{q}^{\mathrm{d}}(\mathfrak{g})$. Let $\mathfrak{w}_{q}^{\mathrm{d}}(\mathfrak{g})^{-}$be the sub-bialgebra of $\mathfrak{w}_{q}^{\mathrm{d}}(\mathfrak{g})$ generated by $F_{i}$, $1 \leq i \leq n$ and $G$. We define a $\mathbb{N}[I]$-algebra gradation on $\mathfrak{w}_{q}^{\mathrm{d}}(\mathfrak{g})^{+}\left(\right.$resp. $\left.\mathfrak{w}_{q}^{\mathrm{d}}(\mathfrak{g})^{-}\right)$such that $E_{j}^{s}$ (resp. $F_{j}^{s}$ ) are homogeneous of degree $s \alpha_{j} \in \mathbb{N}[I]$ for $s \in \mathbb{N}, 1 \leq j \leq n$. We also set $\operatorname{deg} K_{j}=\operatorname{deg} \bar{K}_{j}=\operatorname{deg} J=0$ for all $j$. According to this gradation, $\mathfrak{w}_{q}^{\mathrm{d}}(\mathfrak{g})^{+}$(resp. $\left.\mathfrak{w}_{q}^{\mathrm{d}}(\mathfrak{g})^{-}\right)$is also a graded coalgebra. It is obvious that $\mathfrak{w}_{q}^{\mathrm{d}}(\mathfrak{g})^{+}(1-J) \subset \bar{w}_{q}$ has a basis $\left\{E^{a}(1-J) \mid a \in \mathbb{N}^{\ell_{\mathfrak{o}}}\right\}$. Similarly, $\mathfrak{w}_{q}^{\mathrm{d}}(\mathfrak{g})^{-}(1-J) \subset \bar{w}_{q}$ has a basis $\left\{F^{b}(1-J) \mid b \in \mathbb{N}^{\ell} \overline{\mathfrak{o}}\right\}$.

It is easy to see the elements $E^{a}(1-J), F^{b}(1-J)$ have a gradation $|a|$ and $|b|$, where

$$
\begin{aligned}
& |a|=a_{1} \alpha_{1}+a_{2} s_{i_{1}}\left(\alpha_{i_{2}}\right)+\cdots+a_{t_{\mathfrak{d}}} s_{i_{1}} s_{i_{2}} \cdots s_{i_{t_{\mathfrak{d}}-1}}\left(\alpha_{i_{t_{\mathfrak{d}}}}\right)
\end{aligned}
$$

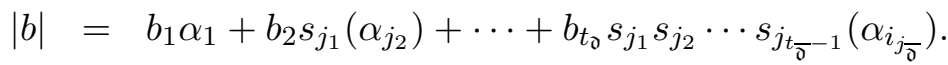

Let

$$
x=\sum_{a \in \mathbb{N}^{\ell_{\mathfrak{o}}}, b \in \mathbb{N}^{\ell} \overline{\mathfrak{\jmath}}} x(a, b) E^{a} F^{b}(1-J) \in \bar{w}_{q},
$$

where $\left\{E^{a} F^{b}(1-J)\right\}$ are linear independent. We have

$$
\begin{aligned}
\Delta(x)= & \sum_{\substack{a \in \mathbb{N} \ell_{0}, b \in \mathbb{N} \\
|s|+|t| \\
|a| \\
|x|+|y|=|b|}} x(a, b) a(s, t) b(x, y) E^{s} F^{x} \bar{K}_{|y|} \otimes E^{t} K_{|s|} F^{y} \\
& -\sum_{\substack{a \in \mathbb{N} \ell_{\mathfrak{o}}, b \in \mathbb{N} \ell \\
|s|+|t|=|a| \\
|x|+|y|=|b|}} x(a, b) a(s, t) b(x, y) E^{s} F^{x} \bar{K}_{|y|} J \otimes E^{t} K_{|s|} F^{y} J .
\end{aligned}
$$


Hence,

$$
\begin{aligned}
& \Delta(x)=\sum_{\substack{a \in \mathbb{N} \ell \\
|,| b \in \mathbb{N} \\
|x|+|y|=|b| \\
\left|a^{\prime}\right|=|a|,|y| \neq 0}} x(a, b) a\left(0, a^{\prime}\right) b(x, y) F^{x} \bar{K}_{|y|} \otimes E^{a^{\prime}} F^{y}(1-J) \\
& +\sum_{\substack{a \in \mathbb{N} \ell_{\mathfrak{d}}, b \in \mathbb{N} \ell \\
|s+|||=\left|\bar{d}^{\prime}\\
\right| b^{\prime}|=| b|,| s \mid \neq 0}} x(a, b) a(s, t) b\left(b^{\prime}, 0\right) E^{s} F^{b^{\prime}}(1-J) \otimes E^{t} K_{|s|} \\
& +\sum_{\substack{a \in \mathbb{N}^{\prime} \ell_{\mathfrak{d}}, b \in \mathbb{N} \\
\left|a^{\prime}\right|=|a| \\
\left|b^{\prime}\right|=|b|}} x(a, b) a\left(0, b^{\prime}\right) b\left(b^{\prime}, 0\right) F^{b^{\prime}} \otimes E^{a^{\prime}} \\
& -\sum_{\substack{a \in \mathbb{N}_{\boldsymbol{\ell}}, b \in \mathbb{N} \ell^{\prime} \bar{\delta} \\
\left|a^{\prime}\right|=|a| \\
\left|b^{\prime}\right|=|b|}} x(a, b) a\left(0, b^{\prime}\right) b\left(b^{\prime}, 0\right) F^{b^{\prime}} J \otimes E^{a^{\prime}} J .
\end{aligned}
$$

On the other hand, by the assumption we have

$$
\begin{aligned}
\Delta(x) & =1 \otimes \sum_{a \in \mathbb{N}^{\ell} \mathfrak{d}, b \in \mathbb{N}^{\ell} \overline{\boldsymbol{d}}} x(a, b) E^{a} F^{b}(1-J)+\sum_{a \in \mathbb{N}^{\ell} \mathfrak{o}, b \in \mathbb{N}^{\ell} \overline{\boldsymbol{d}}} x(a, b) E^{a} F^{b}(1-J) \otimes K_{i} \\
& +a_{x}(1-J) \otimes E_{i} J .
\end{aligned}
$$

Comparing the above identities, we conclude that all $b=0$. Now we can rewrite $x$ as

$$
x=\sum_{a \in \mathbb{N} \ell_{\mathfrak{o}}} x(a) E^{a}(1-J) \in \bar{w}_{q}
$$

and

$$
\begin{aligned}
& \sum_{\substack{a \in \mathbb{N} \ell_{\mathcal{O}} \\
|s|+|t|=|a| \\
|s| \neq 0}} x(a) a(s, t) E^{s}(1-J) \otimes E^{t} K_{|s|}+\sum_{\substack{a \in \mathbb{N}_{0} \\
\left|a^{\prime}\right|=|a|}} x(a) 1 \otimes E^{a^{\prime}}-\sum_{\substack{a \in \mathbb{N}_{0} \\
\left|a^{\prime}\right|=|a|}} x(a) J \otimes E^{a^{\prime}} J \\
& =\sum_{\substack{a \in \mathbb{N} \ell_{\mathbb{D}} \\
|s|+|t|=|a| \\
|s| \neq 0}} x(a) a(s, t) E^{s}(1-J) \otimes E^{t} K_{|s|}+1 \otimes \sum_{\substack{a \in \mathbb{N}^{\prime} \ell_{0} \\
\left|a^{\prime}\right|=|a|}} x(a) E^{a^{\prime}}(1-J) \\
& +\sum_{a \in \mathbb{N} \ell_{\mathfrak{d}}} x(a)(1-J) \otimes E^{a^{\prime}} J \\
& =1 \otimes \sum_{a \in \mathbb{N}^{\ell} \ell_{\mathfrak{d}}} x(a) E^{a}(1-J)+\sum_{a \in \mathbb{N}^{\mathfrak{d}}} x(a) E^{a}(1-J) \otimes K_{i}+a_{x}(1-J) \otimes E_{i} J .
\end{aligned}
$$

Also, comparing the above identity, we conclude that $|a|=\left|a^{\prime}\right|=i$, and hence $x=$ $x(a) E_{i}(1-J)$. It follows that $x(a)=a_{x}$. The argument for $F_{i}$ is similar.

Lemma 5.3 Let $\varphi$ be a Hopf algebra automorphism of $w_{q}$ (the identity is $J$ ). Then there exists a unique way to extend $\varphi$ to $\mathfrak{w}_{q}^{d}(\mathfrak{g})$ such that $\varphi$ is an automorphism of weak Hopf algebra $\mathfrak{w}_{q}^{d}(\mathfrak{g})$. 
Proof. Let $\varphi$ be the automorphism of Hopf algebra $w_{q}$. By Lemma 5.1, the map $\varphi$ is

$$
K_{i} \rightarrow K_{\sigma(i)}, \bar{K}_{i} \rightarrow \bar{K}_{\sigma(i)}, E_{i} J \rightarrow a_{\sigma(i)} E_{\sigma(i)} J, F_{i} J \rightarrow a_{\sigma(i)}^{-1} F_{\sigma(i)} J
$$

for some automorphism $\sigma$ of Dynkin diagram. Assume that $\varphi$ can be extended to $\mathfrak{w}_{q}^{\mathrm{d}}(\mathfrak{g})$ such that $\varphi$ is an automorphism of $\mathfrak{w}_{q}^{\mathrm{d}}(\mathfrak{g})$ as weak Hopf algebras. We have to find a suitable images of $\varphi\left(E_{i}(1-J)\right)$ and $\varphi\left(F_{i}(1-J)\right)$. For example, if $E_{i}$ is type 2, we do nothing since $E_{i}(1-J)=0$. Assume that $E_{i}$ is type 1 and $\varphi\left(E_{i}(1-J)\right)=x$, then $x \neq 0, x \in \bar{w}_{q}$, and

$$
\varphi\left(E_{i}\right)=\varphi\left(E_{i} J+E_{i}(1-J)\right)=a_{\sigma(i)} E_{\sigma(i)} J+x .
$$

Since $\varphi$ is a coalgebra homomorphism, we have

$$
\begin{aligned}
& a_{\sigma(i)} E_{\sigma(i)} J \otimes K_{\sigma(i)}+a_{\sigma(i)} J \otimes E_{\sigma(i)} J+\Delta(x) \\
= & \left(a_{\sigma(i)} E_{\sigma(i)} J+x\right) \otimes K_{\sigma(i)}+1 \otimes\left(a_{\sigma(i)} E_{\sigma(i)} J+x\right) .
\end{aligned}
$$

It follows that

$$
\Delta(x)=x \otimes K_{\sigma(i)}+1 \otimes x+a_{\sigma(i)}(1-J) \otimes E_{\sigma(i)} J .
$$

By Lemma 5.2, $x=a_{\sigma(i)} E_{\sigma(i)}(1-J)$. Similarly, we can get that $\varphi\left(F_{i}(1-J)\right)=a_{\sigma(i)}^{-1} F_{\sigma(i)}(1-$ $J)$ if $F_{i}$ is type 1 , and nothing is done if $F_{i}$ is of type 2 . Of course, $\varphi(1)=1$. The lemma is proved.

For $a=\left(a_{1}, \cdots, a_{n}\right) \in N$, we define a map $\phi_{a}: \mathfrak{w}_{q}^{\mathrm{d}}(\mathfrak{g}) \rightarrow \mathfrak{w}_{q}^{\mathrm{d}}(\mathfrak{g})$ by

$$
\phi_{a}\left(K_{i}\right)=K_{i}, \phi_{a}\left(\bar{K}_{i}\right)=\bar{K}_{i}, \phi_{a}\left(E_{i}\right)=a_{i} E_{i}, \phi_{a}\left(F_{i}\right)=a_{i}^{-1} F_{i} .
$$

It is straightfoward to check that $\phi_{a}$ is a weak Hopf algebra automorphism of $\mathfrak{w}_{q}^{\mathrm{d}}(\mathfrak{g})$. If $\sigma \in H$, then there is an automorphism of weak algebra $\mathfrak{w}_{q}^{d}(\mathfrak{g})$, also denote by $\sigma$, given by

$$
\sigma\left(K_{i}\right)=K_{\sigma(i)}, \sigma\left(\bar{K}_{i}\right)=\bar{K}_{\sigma(i)}, \sigma\left(E_{i}\right)=E_{\sigma(i)}, \sigma\left(F_{i}\right)=F_{\sigma(i)} .
$$

Recall that there is an action of $H$ on $N$ by the rules $\sigma \cdot a=\left(a_{\sigma(1)}, \cdots, a_{\sigma(n)}\right)$ and $\phi_{a} \sigma=\sigma \phi_{\sigma \cdot a}$.

Let $\operatorname{Aut}\left(\mathfrak{w}_{q}^{\mathrm{d}}(\mathfrak{g})\right)$ be the group of automorphisms of weak Hopf algebra $\mathfrak{w}_{q}^{\mathrm{d}}(\mathfrak{g})$. The group $\operatorname{Aut}\left(\mathfrak{w}_{q}^{\mathrm{d}}(\mathfrak{g})\right)$ can be determined by the following theorem.

Theorem 5.4 $\operatorname{Aut}\left(\mathfrak{w}_{q}^{d}(\mathfrak{g})\right)=N \rtimes H$.

Proof. Theorem A in [4] is the key to determine the group of automorphisms of Hopf algebra. But we don't know whether it is true or not for bialgebras (see [5]), we can not directly apply Theorem A to get the result.

Let $\varphi \in \operatorname{Aut}\left(\mathfrak{w}_{q}^{\mathrm{d}}(\mathfrak{g})\right)$, since $\varphi$ sends group-likes to group-likes, we have $\varphi(J)=P^{s}$ for some $s \in \mathbb{Z}^{n}$ by Proposition 4.8. If $s \neq 0$, since $J^{2}=J$ we have $P^{2 s}=P^{s}$, hence $P^{s}=J$ it follows that $P^{s} J=J$. It is a contradiction for Theorem 4.6. Therefore, $s=0$ and $\varphi(J)=J$.

According to Proposition $4.2, \mathfrak{w}_{q}^{\mathrm{d}}(\mathfrak{g})=w_{q} \oplus \bar{w}_{q}$ and $w_{q} \cong U_{q}(\mathfrak{g})$ as Hopf algebras, where the notations $w_{q}$ and $\bar{w}_{q}$ as before. 
Let $\operatorname{inj}_{q}: w_{q} \rightarrow \mathfrak{w}_{q}^{\mathrm{d}}(\mathfrak{g})$ be the inclusion defined by

$$
J \rightarrow J, E_{i} J \rightarrow E_{i} J, F_{i} J \rightarrow F_{i} J, K_{i} \rightarrow K_{i}, \overline{K_{i}} \rightarrow \bar{K}_{i},
$$

and then extend it by linearity. It is easy to see that $i n j_{q}$ is a weak Hopf algebra injection by Proposition 4.2. Let $\varphi \in \operatorname{Aut}\left(\mathfrak{w}_{q}^{\mathrm{d}}(\mathfrak{g})\right)$, we see that $w_{q}=\operatorname{im}\left(\varphi \circ i n j_{q}\right)$ since $\varphi(J)=J$. This implies that $\varphi \circ \operatorname{inj}_{q}$ is an automorphism of Hopf algebra $w_{q}$ and $\varphi \circ \operatorname{inj}_{q} \in N \rtimes H$ by Theorem 5.1. That is, the map $\left.\varphi\right|_{w_{q}}$ is

$$
K_{i} \rightarrow K_{\sigma(i)}, \bar{K}_{i} \rightarrow \bar{K}_{\sigma(i)}, E_{i} J \rightarrow a_{\sigma(i)} E_{\sigma(i)} J, F_{i} J \rightarrow a_{\sigma(i)}^{-1} F_{\sigma(i)} J .
$$

This implies that $\varphi$ is

$$
K_{i} \rightarrow K_{\sigma(i)}, \bar{K}_{i} \rightarrow \bar{K}_{\sigma(i)}, E_{i} \rightarrow a_{\sigma(i)} E_{\sigma(i)}, F_{i} \rightarrow a_{\sigma(i)}^{-1} F_{\sigma(i)}
$$

by Lemma 5.2. Hence $\varphi \in N \rtimes H$ and $\operatorname{Aut}\left(\mathfrak{w}_{q}^{\mathrm{d}}(\mathfrak{g})\right) \subseteq N \rtimes H$. On the other hand, it is obvious that $N \rtimes H \subseteq \operatorname{Aut}\left(\mathfrak{w}_{q}^{\mathrm{d}}(\mathfrak{g})\right)$. The proof is completed.

The more interesting problem is to determine the algebraic group of $\mathfrak{w}_{q}^{\mathrm{d}}(\mathfrak{g})$. It is mentioned that [1] contributed to determine $\operatorname{Aut}_{A l g}\left(U_{q}^{+}(\mathfrak{g})\right)$. A basic idea to approach the

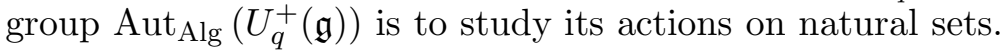

\section{Acknowledgment}

The author is grateful to the referee for his/her helpful comments.

\section{References}

[1] N. Andruskiewitsch and F. Dumas, On the automorphisms of $U_{q}^{+}(\mathfrak{g})$, arXiv.math.QA/0301066.

[2] N. Aizawa and P. S. Isaac, Weak Hopf algebras corresponding to $U_{q}\left[s l_{n}\right]$, J. Math. Phys., 44(11), 2003, 5250-5267.

[3] G. Böhm, F. Nill, and K. Szlachányi, Weak Hopf algebras I. Integral theory and $C^{*}$-structure, J. Algebra 221, 385-438(1999).

[4] W. Chin and I. M. Musson, The coradical filtration for quantized enveloping algebras, J. London Math. Soc. (2) 53(1996) 50-62.

[5] W. Chin and I. M. Musson, Corrigenda, the coradical filtration for quantized enveloping algebras, J. London Math. Soc. (2) 61(2000) 319-320.

[6] J. A. Green, W. D. Nicols, and E. J. Taft, Left Hopf algebras, J. Algebra 65 (1980), 399-411.

[7] T. Hayashi, An algebra related to the fusion rules of Wess-Zumino-Witten models, Lett. Math Phys. 22 (1991), 291-296. 
[8] J. Humphreys, Introduction to Lie Algebras and Representation Theory, SpringerVerlag. GTM. 9, 1972.

[9] C. Kassel, Quantum Groups, Springer-Verlag, New York, 1995.

[10] J. C. Jantzen, Lectures on Quantum Group, Amer Math Society, GSM, Vol. 6, 1995.

[11] F. Li, Weak Hopf algebras and new solutions of Yang-Baxter equation, J. Algebra 208 (1998), 72-100.

[12] F. Li, S. Duplij, Weak Hopf algebras and singular solutions of quantum Yang-Baxter equation, Commun. Math. Phys. 225(2002), 191-217.

[13] G. Lusztig, Introduction to Quantum Groups Progress in Math., Birkhauser Vol. 110, 1993.

[14] S. Montgomery, Hopf Algebras and Their Actions on Rings, CBMS, Lecture in Math. 82, AMS, Providence, RI, 1993.

[15] E. Müller, The coradical filtration of $U_{q}(\mathfrak{g})$ at roots of unity, Comm. in Algebra, 28(2000),1029-1044.

[16] W. D. Nichols and E. J. Taft, The left antipodes of a left Hopf algebra, Contemp. Math. ,13, Amer. Math. Soc., Providence, 1982.

[17] M. E. Sweedler, Hopf Algebras, Benjamin, New York, 1969.

[18] E. Twietmeyer, Real forms of $U_{q}(\mathfrak{g})$, Lett. Math. Phys. 24(1992) 49-58.

[19] T. Yamanouchi, Duality for generalized Kac algebras and a characterization of finite groupoid algebras, J. Algebra 163, 9-50(1994). 